\title{
MAGNETIC CHARACTERISTICS OF THE SYSTEM OF EXCHANGE COUPLED LAYERS
}

\author{
A. UrbaniaK-KuCharczyK aNd S. MaChowski \\ Department of Solid State Physics, University of Eódź \\ Pomorska 149/153, 90-236 Łódź, Poland
}

\begin{abstract}
Using the Green function method it is shown that the deviation of the Curie temperature from its value calculated for continuous layers is related to the absolute value of the interlayer coupling parameter. The dependence of spin wave stiffness parameter on the interlayer exchange coupling is also found.
\end{abstract}

PACS numbers: $75.70 . \mathrm{Ak}, 75.30 .-\mathrm{m}, 75.40 . \mathrm{Cx}$

\section{Introduction}

The interlayer exchange coupling of ferromagnetic transition metal layers through non-magnetic spacer has been intensively studied over the last decade (see, e.g. [1-6]). In comparison, little work was devoted to the magnetic characteristics of such systems. An oscillatory interlayer exchange coupling observed in many multilayered systems can be understood as an interference effect of electron waves partially reflected at each interface with spin dependent reflection coefficients [7]. Therefore, one can expect the magnetic properties, which are related to the interlayer exchange coupling parameter, to oscillate as a function of the spacer thickness.

However, relatively few results have been reported concerning experimental investigations of basic magnetic properties of exchange coupled layers. Oscillations of the magnetic anisotropy and the Kerr rotation angle in magnetic films covered by non-magnetic overlayers have been reported $[8,9]$. It has been also experimentally found that the Curie temperature, ground state magnetic moment, and spin wave stiffness parameter are the oscillatory functions of the thickness of non-magnetic layer [10]. Moreover, in the recent paper the onset of long-range order in exchange coupled epitaxial monolayers is investigated by temperature dependent susceptibility and element resolved magnetization measurements [11]. 


\section{Method of calculation}

In order to investigate the influence of interlayer exchange coupling we consider the system of two thin homogeneous ferromagnetic layers separated by non-magnetic spacer. The thickness of ferromagnetic layer is equal to $N$ and $M$ monoatomic planes, respectively. The system is described by the Heisenberg Hamiltonian in the following form:

$$
\mathcal{H}=-\frac{1}{2} \sum_{\nu j, \nu^{\prime} j^{\prime}} J_{\nu j \nu^{\prime} j^{\prime}} S_{\nu j} S_{\nu^{\prime} j^{\prime}}-g \mu_{\mathrm{B}} H \sum_{\nu j} S_{\nu j}^{z}-\frac{1}{2} \sum_{\nu j^{\prime} j^{\prime}} A_{\nu j \nu^{\prime} j^{\prime}} S_{\nu j^{\prime}}^{z} S_{\nu^{\prime} j^{\prime}}^{z}
$$

where $\nu \in(1, N+M)$ denotes the number of monoatomic planes and $j$ defines the position of a lattice point in the plane $\nu$. The exchange parameter $J_{\nu j \nu^{\prime} j^{\prime}}$ is assumed as

$$
J_{\nu j \nu^{\prime} j^{\prime}}= \begin{cases}J_{0} & \text { for } \quad \nu, \nu^{\prime} \in(1, N-1) \text { and }(N+2, M), \\ J_{12} & \text { for } \quad \nu, \nu^{\prime} \in N, N+1,\end{cases}
$$

where $J_{0}$ is the exchange integral for homogeneous medium and $J_{12}$ denotes the exchange parameter between spins belonging to interface layers in different magnetic sublayers. $J_{12}$ which describes the coupling through the interface can be either positive (ferromagnetic coupling) or negative (antiferromagnetic coupling) in dependence on the spacer thickness and its orientation [12]. The anisotropy parameter $A_{\nu j, \nu^{\prime} j^{\prime}}$ consists of the uniaxial volume anisotropy parameter $A$, the surface anisotropy parameter $A_{\mathrm{S}}$, and interface anisotropy parameter $A_{\mathrm{I}}$, namely

$$
A_{\nu j \nu^{\prime} j^{\prime}}=A+A_{\mathrm{S}}\left(\delta_{1,2}+\delta_{M-1, M}\right)+A_{\mathrm{I}} \delta_{(N, N+1)} .
$$

Parameters $A_{\mathrm{S}}$ and $A_{\mathrm{I}}$ are allowed to be either of easy axis or easy plane type.

Following the usual procedures of the Green function method [13, 14] we write the equation for the magnetization in $\nu$-th layer

where

$$
\left\langle S_{\nu}^{z}\right\rangle=S\left[\frac{\mathrm{d}}{\mathrm{d} r}\left\langle\exp \left(r S_{\nu}^{z}\right)\right\rangle\right]_{r=0},
$$

$$
\left\langle\exp \left(r S_{\nu}^{z}\right)\right\rangle=\frac{\varphi_{\nu}^{2 S+1} \mathrm{e}^{-r s}+\left(1+\varphi_{\nu}\right)^{2 S+1} \mathrm{e}^{r(S+1)}}{\left[\varphi_{\nu}^{2 S-1}-\left(1+\varphi_{\nu}\right)^{2 S+1}\right]\left[\left(1+\varphi_{\nu}\right) \mathrm{e}^{r}-\varphi_{\nu}\right]}
$$

with

$$
\varphi_{\nu}=\frac{1}{n} \sum_{h} \sum_{i=1}^{M} \frac{b_{\nu}^{2}\left(k_{i}\right)}{\exp \left[\frac{E\left(k_{i}, h\right)}{k_{\mathrm{B}} T}\right]-1}
$$

where the form of $E\left(k_{i}, h\right)$ in the first order random phase approximation (RPA) and the system of equations determining $k_{i}$ are given in [15].

\section{Numerical results}

To calculate the magnetic characteristics of exchange coupled layers described by Hamiltonian (1) we take into account the theoretical dependence of $J_{12}$ on the spacer thickness $d$ (in number of monoatomic planes) obtained on the basis of extended Zener model for the fcc spacer [6]. In the considered model the coupling between spins belonging to the outmost atomic planes via the conduction 
electrons in the spacer has been calculated within the Green function formalism in the Hartree-Fock approximation. It has been shown that the interlayer exchange parameter $J_{12}$ obtained using the interaction parameters corresponding to $\mathrm{Cu}$, is of an oscillatory character as a function of the spacer thickness and depends on the orientation of the spacer.

To investigate the influence of exchange coupling on the Curie temperature we assume the uniaxial volume anisotropy parameter equal to $10^{-4} J_{0}$, while $A_{\mathrm{S}}=A_{\mathrm{I}}=0$. Reduced Curie temperature $y=T_{\mathrm{C}}\left(2 N, J_{12}\right) / T_{\mathrm{C}}\left(2 N, J_{12}=1\right)$ of magnetic bilayer plotted as a function of parameter $f=\left|J_{12} / J_{0}\right|$ calculated - on the basis of the method described in Sec. 2 is presented in Figs. 1 and 2 for two orientations of fcc spacer. Thicknesses of both constituent layers are equal to $5 \mathrm{ML}$.

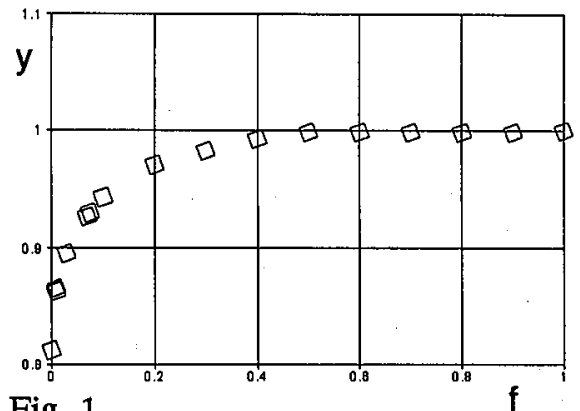

Fig. 1

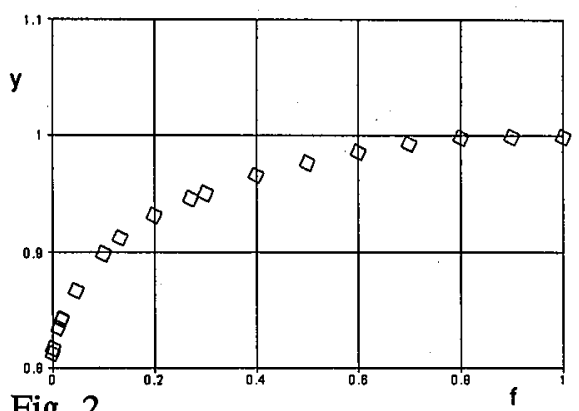

Fig. 2

Fig. 1. The dependence of reduced Curie temperature $y$ on the interlayer coupling parameter $f$ for ferromagnetically coupled layers separated by fcc (100) non-magnetic spacer. $N=M=5$.

Fig. 2. The dependence of reduced Curie temperature $y$ on the interlayer coupling parameter $f$ for antiferromagnetically coupled layers separated by fcc (111) non-magnetic spacer. $N=M=5$.

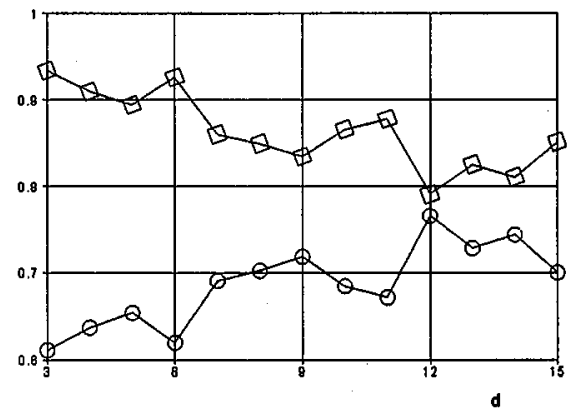

Fig. 3. The reduced Curie temperature $y-$ upper plot and spin wave parameter $B$ (in $\left.10^{-4} \mathrm{~K}^{-3 / 2}\right)$ - lower plot, as a function of spacer thickness in exchange coupled layers separated by fcc (100) spacer. $N=M=4$. 
Figure 3 presents the comparison of the behavior of the Curie temperature and spin wave parameter $B$ calculated as a function of the spacer thickness $d$. The results obtained describe in qualitative way experimental findings [10]. The presented model can be easily developed for multicomponent systems.

\section{References}

[1] J. Unguris, R.J. Cellota, D.T. Pierce, Phys. Rev. Lett. 67, 140 (1991).

[2] S.S.P. Parkin, R. Bhandra, K.P. Roche, Phys. Rev. Lett. 66, 2152 (1991).

[3] P. Bruno, C. Chappert, Phys. Rev. B 46, 261 (1992).

[4] J. Barnaś, J. Magn. Magn. Mater. 111, L215 (1992).

[5] Q.Y. Jin, Y.B. Xu, H.R. Zhai, C. Hu, M. Lu, Q.S. Bie, Phys. Rev. Lett. 72, 768 (1994).

[6] A. Urbaniak-Kucharczyk, Phys. Status Solidi B 188, 795 (1995).

[7] P. Bruno, Phys. Rev. 52, 411 (1995).

[8] B.N. Engel, M.H. Wiedemann, R.A. Van Leeuwen, C.M. Falco, Phys. Rev. B 48, 9894 (1993).

[9] R. Mégy, A. Bounouh, Y. Suzuki, P. Beauvilloin, P. Bruno, C. Chappert, B. Lecuyer, P. Veillet, Phys. Rev. B 51, 5586 (1995).

[10] G. Bayreuther, F. Bensch, V. Kotler, J. Appl. Phys. 79, 4509 (1996).

[11] U. Bovensiepen, F. Wilhelm, P. Srivasta va, P. Poulopoulos, M. Farle, A. Ney, K. Barberschke, Phys. Rev. Lett. 81, 2368 (1998).

[12] P. Bruno, C. Chappert, Phys. Rev. Lett. 67, 1602, E2592 (1991).

[13] S.W. Tyablikov, Metody kvantovoi teorii magnetizma, Izd. Nauka, Moskva 1965 (in Russian).

[14] H.B. Callen, Phys. Rev. 130, 890 (1963).

[15] A. Urbaniak-Kucharczyk, Phys. Status Solidi B 203, 195 (1997). 